\title{
RANGES OF LYAPUNOV TRANSFORMATIONS FOR HILBERT SPACE
}

\author{
by J. KYLE
}

(Received 30 September, 1976; revised 28 October, 1976)

1. Introduction. Interest in the ranges of Lyapunov transformations began with Taussky in [5]. Recently in a series of papers, Loewy has studied the ranges of Lyapunov transformations on matrices. In particular in [2] and [3], the following result was obtained.

Theorem. Let $A, B \in \mathbb{C}^{n, n}$ and suppose that $L_{\mathrm{A}}$ is invertible. Then the following are equivalent.

(i) $B=(\alpha I+i \beta A)(\gamma A+i \delta I)^{-1}$ for some real scalars with $\alpha \gamma+\beta \delta=1$.

(ii) $L_{A}(\operatorname{PSD}(n))=L_{B}(\operatorname{PSD}(n))$.

Here $L_{A}$ is the Lyapunov transformation corresponding to $A$ i.e. $L_{A}(X)=A X+X A^{*}$, and $\operatorname{PSD}(n)$ denotes the set of all positive semi-definite $n \times n$ matrices.

In this short note we shall obtain a generalization of the above result to the case when $A$ and $B$ are bounded linear operators on any Hilbert space.

2. Mappings which preserve the positive cone. Let $H$ be any Hilbert space and let $L(H)$ denote the set of all bounded linear operators on $H . L(H)^{\text {sa }}$ and $L(H)^{+}$will denote the self-adjoint and positive parts of $L(H)$ respectively.

2.1 Lemma. Suppose $\alpha$ is an invertible bounded linear mapping of $L(H)$ onto itself which takes $L(H)^{+}$onto itself, and maps $I$ to $I$. Then $\alpha$ is either a ${ }^{*}$-automorphism of $L(H)$ or $a^{*}$-anti-automorphism of $L(H)$. $\|H\|$.

Proof. Let $H \geqslant 0$. Then $\|H\| I-H \geqslant 0$, so that $\alpha(H) \leqslant \alpha(\|H\| I)=\|H\| I$. Thus $\|\alpha(H)\| \leqslant$

The same argument applied to $\alpha^{-1}$ shows that $\|H\| \leqslant\|\alpha(H)\|$. Hence $\alpha$ is isometric on $L(H)^{+}$. $\alpha$ therefore preserves the extreme points of the positive part of the unit ball, i.e. $\alpha$ maps projections onto projections.

From here we may argue as Kadison in [1] to show that $\alpha$ is (in the terminology of [1]) a $C^{*}$-isomorphism of $L(H)$. The proof is completed by noting that it is shown also in [1] that such a map on $L(H)$ must be either a *-automorphism or a *-anti-automorphism of $L(H)$.

2.2 Lemma. Suppose $L_{A}$ and $L_{B}$ are two invertible Lyapunov transformations of $L(H)$ and that $L_{A}\left(L(H)^{+}\right)=L_{B}\left(L(H)^{+}\right)$. Then there is some non-singular $V$ in $L(H)$ such that $L_{A}^{-1} L_{B}(X)=V X V^{*}$ for all $X$ in $L(H)$.

Proof. $L_{A}^{-1} L_{B}\left(L(H)^{+}\right)=L(H)^{+}$. Let $T \in L(H)^{+}$be such that $L_{A}^{-1} L_{B}(T)=I$. Then $\|T\|^{-1} T \leqslant I$, so that $\|T\|^{-1} I=L_{A}^{-1} L_{B}\left(\|T\|^{-1} T\right) \leqslant L_{A}^{-1} L_{B}(I)$. Thus $L_{A}^{-1} L_{B}(I)$ is invertible and equals $S^{2}$ for some non-singular $S$ in $L(H)^{\text {sa }}$.

Glasgow Math. J. 19 (1978) 99-101 
Let $\alpha(X)=S^{-1} L_{A}^{-1} L_{B}(X) S^{-1}$. Then $\alpha$ satisifies the conditions of Lemma 2.1 and so is either a ${ }^{*}$-automorphism or a ${ }^{*}$-anti-automorphism.

If $\alpha$ is a *automorphism, we can find a unitary $U$ in $L(H)$ such that $\alpha(X)=U X U^{*}$ (see [4 Ch. 4], for example). Taking $V=S U$ gives the required result.

Now if $\alpha$ is a ${ }^{*}$-anti-automorphism of $L(H)$, by the same token we can find a conjugate linear isomorphism $U$ of $H$ onto itself such that $\alpha(X)=U X^{*} U^{-1}$ for all $X$ in $L(H)$. Thus $L_{B}(X)=L_{A}\left(S U X^{*} U^{-1} S\right)$. Now if $X$ is given by $X \xi=\langle\xi, \sigma\rangle \tau$ (for some $\sigma, \tau$ in $H), X^{*} \xi=\langle\xi, \tau\rangle \sigma$ and evaluating both sides at $\sigma$ gives

$$
\langle\sigma, \sigma\rangle B \tau+\left\langle B^{*} \sigma, \sigma\right\rangle \tau=\left\langle U^{-1} S \sigma, \tau\right\rangle A S U \sigma+\left\langle U^{-1} S A^{*} \sigma, \tau\right\rangle S U \sigma .
$$

The L.H.S. is linear in $\tau$, the R.H.S. is conjugate linear in $\tau$. Thus both sides are identically zero, from which one can deduce that $A$ and $B$ are both scalar multiples of $I$. $S$ can then be chosen to be a positive multiple of $I$, so that $\alpha$ is the identity map. This contradicts the assumption that $\alpha$ is an anti-automorphism.

3. Main result. We first prove:

3.1 LeMMA. $L_{A}=L_{B}$ if and only if $B=A+i \lambda I$ for some $\lambda \in \mathbb{R}$.

Proof. "if" is easy. Suppose $A=A_{1}+i A_{2}$ (with $A_{i}$ self-adjoint) and suppose that $L_{A}=0$. Then $0=L_{A}(I)=2 A_{1}$, and so $A_{1}=0$. Hence, for all $X$ in $L(H), 0=L_{A}(X)=$ $i\left(A_{2} X-X A_{2}\right)$ and so $A_{2}=\lambda I$ for some real $\lambda$.

3.2 TheOREM. Suppose $L_{A}$ and $L_{B}$ are two non-singular Lyapunov transformations. Then the following are equivalent.

(i) $B=(\alpha I+i \beta A)(\gamma A+i \delta I)^{-1}$ for some real $\alpha, \beta, \gamma, \delta$ with $\alpha \gamma+\beta \delta=1$.

(ii) $L_{A}\left(L(H)^{+}\right)=L_{B}\left(L(H)^{+}\right)$.

Proof. (i) $\Rightarrow$ (ii). As in [2] and [3] we can show that (i) implies that either $L_{B}$ is a positive multiple of $L_{A}$ or a positive multiple of $L_{(A+i \lambda I)^{-1}}$ for real $\lambda$. From this (ii) follows easily.

(ii) $\Rightarrow$ (i). Using Lemma 2.2, we can find some non-singular $V$ in $L(H)$ such that $B X+X B^{*}=A V X V^{*}+V X V^{*} A^{*}$ for all $A$ in $L(H)$. If $X$ is given by $X \xi=\langle\xi, \sigma\rangle \tau$ where $\sigma, \tau$ are arbitrary vectors of norm one, we get

$$
B \tau+\left\langle B^{*} \sigma, \sigma\right\rangle \tau=\left\langle V^{*} \sigma, \sigma\right\rangle A V \tau+\left\langle V^{*} A^{*} \sigma, \sigma\right\rangle V \tau
$$

Choosing two values of $\sigma$ and eliminating $B \tau$ from (1) we see that

$$
V=(\gamma A+i \delta)^{-1}
$$

for some scalars $\gamma$ and $\delta$. If $\gamma=0, V$ is a multiple of $I$ and so $L_{B}$ is a multiple (which must be positive) of $L_{A}$. Lemma 3.1 then allows us to display $B$ in the form (i). We shall therefore assume that $\gamma \neq 0$. Multiplying $V$ by some scalar of unit modulus leaves the equations unaltered, but allows us to assume that $\gamma$ is real. Further, using (1) with $\sigma=\tau$ gives

$$
2 \operatorname{Re}\langle B \sigma, \sigma\rangle=2 \gamma^{-1} \operatorname{Re}\langle V \sigma, \sigma\rangle+i \gamma^{-1}(\bar{\delta}-\delta)|\langle V \sigma, \sigma\rangle|^{2}
$$

and so $\delta=\bar{\delta}$ as required. 
Returning once more to (1), we see that $B+\lambda I=\nu A V+\mu V$ for some scalars $\lambda, \mu, \nu$. This may be rewritten as $B V^{-1}=(\alpha I+i \beta A)$ for some scalars $\alpha, \beta$. Using the original equation we get $B V^{-1} X V^{*-1}+V^{-1} X V^{*-1} B^{*}=A X+X A^{*}$ for all $X$ in $L(H)$ which reduces to

$$
(\bar{\alpha} \gamma+\beta \delta-1) A X+(\alpha \gamma+\delta \bar{\beta}-1) X A^{*}=i(\gamma \bar{\beta}-\beta \gamma) A X A^{*} .
$$

This shows that $(\gamma \vec{\beta}-\beta \gamma)=0$, and so $\beta \in \mathbb{R}$, and gives $\gamma L_{\alpha A}+(\beta \delta-1) L_{A}=0$. Hence, by Lemma 3.1, $\bar{\alpha} A=-\gamma^{-1}(\beta \delta-1) A+i \lambda I$, for some $\lambda \in \mathbb{R}$. Thus $\alpha=-\gamma^{-1}(\beta \delta-1)$ as required, or $A$ is a multiple of $I$. In this case either $L_{B}$ or $-L_{B}$ maps $L(H)^{+}$onto $L(H)^{+}$ and so (as in \$2) $L_{B}(X)=U X U^{*}$ for some $U$ in $L(H)$. Arguing as above shows that $U$ is a multiple of $I$, and so (by Lemma 3.1) also is $B$. This completes the proof.

3.3 Remarks. For finite dimensional $H, L_{B}$ is non-singular whenever $L_{A}$ is, and so Theorem 3.2 gives Loewy's theorem. Essentially, this is because any linear mapping taking $\operatorname{PSD}(n)$ onto $\operatorname{PSD}(n)$ must be non-singular. This may be false, however, in general. For example, when $H=\ell^{2}\left(Z^{+}\right)$and $U$ is the unilateral shift, the mapping $\alpha(X)=U^{*} X U$ takes $L(H)^{+}$onto $L(H)^{+}$yet has no inverse.

The author would like to thank the referee for making some helpful comments in the preparation of this paper.

\section{REFERENCES}

1. R. V. Kadison, Isometries of operator algebras, Ann. of Math. 54 (1951), 325-338. $112-118$

2. R. Loewy, On ranges of Lyapunov transformations IV, Glasgow Math. J. 17 (1976),

3. R. Loewy, On ranges of Lyapunov transformation III, S.I.A.M. J. Appl. Math. 30 (1976), 687-702.

4. S. Sakai, $C^{*}$-algebras and $W^{*}$-algebras (Springer-Verlag, 1971).

5. O. Taussky, Matrix theory research problem, Bull. Amer. Math. Soc. 71 (1965), 711.

DePARTMENT OF MATHEMATICS, UNIVERSITY OF TECHNOLOGY, LOUGHBOROUGH.
Present address

Department of Pure Mathematics, UNIVERSITY OF BIRMINGHAM, P.O. Box 363

BirminghaM B15 2TT. 